\title{
Momentum transfer to fluffy dust aggregates from stellar winds
}

\author{
T. Minato ${ }^{1}$, M. Köhler ${ }^{1}$, H. Kimura ${ }^{2}$, I. Mann ${ }^{1}$, and T. Yamamoto ${ }^{2}$ \\ 1 Institut für Planetologie, Westfälische Wilhelms-Universität, Wilhelm-Klemm-Str. 10, 48149 Münster, Germany \\ 2 Institute of Low Temperature Science, Hokkaido University, Kita-ku Kita-19 Nishi-8, Sapporo 060-0819, Japan \\ e-mail: ty@lowtem.hokudai.ac.jp
}

Received 25 December 2005 / Accepted 27 January 2006

\section{ABSTRACT}

\begin{abstract}
Aims. Dust-plasma interaction may play a dominant role in the dynamics of dust particles around young main-sequence stars. Circumstellar dust is expected to be an aggregate consisting of small grains. The momentum transfer cross section (MTCS) for an aggregate is a key quantity for determining the lifetimes of circumstellar dust disks.

Methods. We formulate the MTCS of fluffy dust aggregates and propose an algorithm for computing the MTCS. We compare the magnitude of the plasma and photon Poynting-Roberson effects (PR effects).

Results. We find an empirical formula that approximates numerical results well for the MTCSs. A comparison of the magnitudes of the PR effects shows that the lifetime of debris dust around young main-sequence stars is shorter by orders of magnitude than the lifetime estimated by considering the photon PR effect alone. Brief discussion is given on the plasma PR effect for dust debris disks around young main-sequence stars.
\end{abstract}

Key words. stars: winds, outflows - solar wind - meteors, meteroids - interplanetary medium - Kuiper Belt - circumstellar matter

\section{Introduction}

Dust particles orbiting a star spiral into the star owing to the drag by stellar photons that are absorbed and scattered by the dust. This is called the Poynting-Robertson effect (PR effect). Stellar winds striking dust also decrease its orbital angular momentum, which we call the plasma PR effect analogous to the photon PR effect. Both photon and plasma PR effects play a role in limiting the lifetime of dust around main-sequence stars. An evaluation of the plasma PR lifetimes requires knowledge of interaction between dust and stellar wind ions, in other words, the momentum transfer cross section (MTCS). Stellar wind ions are not perfectly absorbed by a dust particle, but penetrate through it if their momentum is sufficiently large. This reduces the MTCS of the dust as demonstrated by Minato et al. (2004) who take penetration of stellar wind ions into account for a study of the plasma PR effect. Clearly, realistic estimates of the MTCS are the key to better understanding the lifetimes of dust particles (see Plavchan et al. 2005).

In the previous studies of the plasma PR effect, dust is assumed for simplicity to be a spherical grain (Burns et al. 1979; Mukai \& Yamamoto 1982; Gustafson 1994; Fahr et al. 1995; Minato et al. 2004). However, cometary dust is often assumed to be fluffy aggregates consisting of submicron grains. These aggregates are in fact observed as interplanetary dust particles (e.g., Brownlee 1978). One expects that dust in the disks around main-sequence stars also has a fluffy structure, which increases the geometric cross sections of particles compared with a spherical dust of the same volume. In consequence, this may increase their MTCSs. If this is the case, the orbital angular momentum of dust aggregates is more efficiently lost compared to that of spherical dust particles. The importance of the plasma PR effect also depends on the stellar-wind parameters. Recently, Wood et al. (2002) have shown that the stellar winds of solar-like stars become weaker with the age of the star. Therefore, the plasma
PR effect may even play a dominant role in determining the lifetimes of dust particles around young main-sequence stars (Mukai et al. 2004).

In this paper, we study the plasma PR effect for dust aggregates and its consequences on their lifetimes in disks around main-sequence stars. We first derive the MTCSs for dust aggregates and then calculate the stellar wind pressure and plasma PR drag forces to compare with the radiation pressure and photon PR drag forces. We finally discuss the results in terms of lifetimes for dust around main-sequence stars with a variety of ages.

\section{Formulation of the MTCS of a dust aggregate}

The winds of solar-like stars typically attain a kinetic energy $E$ of $\mathrm{keV} /$ nucleon. In this energy range, the stopping power of protons and $\alpha$ particles are proportional to $E^{1 / 2}$ (Fermi \& Teller 1947; Lindhard et al. 1963; Sigmund \& Schinner 2002). In this case, the momentum $p$ after passing thickness $\zeta$ in matter is given in Minato et al. (2004) as

$$
\frac{p_{0}-p}{p_{0}}=\frac{\zeta}{l\left(p_{0}\right)}
$$

where $p_{0}$ is the initial momentum. The projected range $l\left(p_{0}\right)$ of an ion having initial energy $E_{0}=p_{0}^{2} /(2 m)$ and mass $m$ is defined by

$l\left(p_{0}\right)=\int_{p=0}^{p_{0}} \frac{\mathrm{d} E}{-\mathrm{d} E / \mathrm{d} x}=\frac{2 E_{0}}{\left.(-\mathrm{d} E / \mathrm{d} x)\right|_{E=E_{0}}}$

We consider an aggregate consisting of $N$ identical spherical grains of radius $a$. The geometry of the aggregate is determined by giving the position vectors $\boldsymbol{r}_{i}(i=1, \ldots, N)$ of the centers of each grain composing the aggregate. We take the $z$ axis that is 

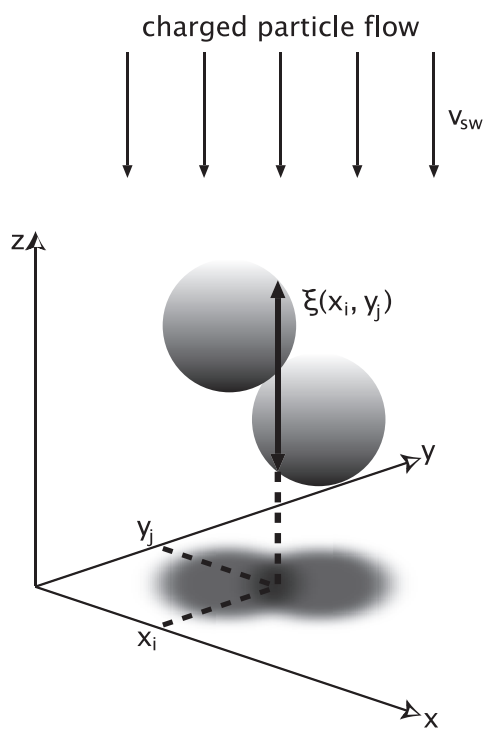

Fig. 1. A schematic view of a dust aggregate irradiated by stellar wind ions. The shadow on the $x y$ plane expresses the geometrical cross section of the aggregate.

antiparallel to a flow of stellar wind ions (see Fig. 1). An important quantity in calculating the MTCS of a dust aggregate from stellar winds is the thickness of the aggregate along a path of stellar wind ions. The thickness is defined by the total length that the ions pass through grains composing the aggregate, and is given by

$\xi(x, y)=\sum_{i=1}^{N} \int_{-\infty}^{\infty} \mathrm{d} z U\left(a-\left|\boldsymbol{r}-\boldsymbol{r}_{i}\right|\right)$

where $U(x)$ is the step function defined by $U(x)=1$ for $x>0$ and $U(x)=0$ for $x<0$. The thickness varies with the impact parameters of the ions. The distribution function of the thickness, $f(\zeta)$, is defined to be a projected area beneath which depth $\xi$ is in the projected range of $\zeta<\xi(x, y)<\zeta+\mathrm{d} \zeta$ :

$f(\zeta) \mathrm{d} \zeta=\mathrm{d} \zeta \int \mathrm{d} S \delta(\zeta-\xi(x, y)+\epsilon)$

where $\delta(x)=\mathrm{d} U(x) / \mathrm{d} x$ is the delta function and $\epsilon$ infinitesimally small positive number. Here, $\xi$ is replaced by $\xi-\epsilon$ to eliminate the contribution of the surface element $\mathrm{d} S=\mathrm{d} x \mathrm{~d} y$ outside the aggregate to the integral. The zeroth and first moments of $f(\zeta)$ lead to the geometrical cross section $S_{z}$ projected onto the $x y$ plane and the volume $V$ of the aggregate, respectively:

$S_{z}=\int_{0}^{\infty} \mathrm{d} \zeta f(\zeta), \quad V=\int_{0}^{\infty} \mathrm{d} \zeta \zeta f(\zeta)$

Using the thickness distribution function $f(\zeta)$, the MTCS of the aggregate is given by

$$
\begin{aligned}
C_{\mathrm{sw}} & =\frac{1}{l\left(p_{0}\right)} \int_{0}^{\infty} \mathrm{d} \zeta f(\zeta) \min \left\{\zeta, l\left(p_{0}\right)\right\} \\
& =\frac{1}{l\left(p_{0}\right)} \int_{0}^{l\left(p_{0}\right)} \mathrm{d} \zeta \zeta f(\zeta)+\int_{l\left(p_{0}\right)}^{\infty} \mathrm{d} \zeta f(\zeta) .
\end{aligned}
$$

The first term on the right-hand side indicates the volume of the region where the incident ions pass through the aggregate, and the second term the geometrical cross section of the region where the incident particles are absorbed. The MTCS $C_{\mathrm{sw}}$ has the following characteristics. First, if the thickness of the aggregate is so small that the projected range of the ions is larger than the thickness along any line of sight, then $C_{\mathrm{sw}}$ reduces to $C_{\text {sw }}=V / l\left(p_{0}\right)$. Namely, the cross section is proportional to the volume of the aggregate and is irrelevant to its shape and structure. Second, if the thickness is so large that the thickness along any line of sight is larger than the projected range except the edge of the aggregate, then $C_{\mathrm{sw}}$ reduces to the geometrical cross section $S_{z}$. Note that these limits correspond to the Rayleigh limit and to the geometrical-optics limit in light scattering.

It is easy to see that in the case of a spherical grain Eq. (6) leads to the MTCS given in our previous study (Minato et al. 2004). We take the cylindrical coordinates in which the origin is placed at the center of the grain and the $z$ axis is parallel to the wind velocity. The thickness of the grain along a path of a stellar wind ion incident with impact parameter $\rho$ is given by

$\xi= \begin{cases}2 \sqrt{a^{2}-\rho^{2}} & (0 \leq \rho \leq a), \\ 0 & (\rho>a) .\end{cases}$

The distribution function of the thickness is calculated from Eq. (4) to be

$f(\zeta) \mathrm{d} \zeta=2 \pi \mathrm{d} \zeta \int_{0}^{a} \mathrm{~d} \rho \rho \delta\left(\zeta-2 \sqrt{a^{2}-\rho^{2}}+\epsilon\right)=\frac{\pi}{2} \zeta \mathrm{d} \zeta$

for $\zeta<2 a$, and $f(\zeta)=0$ for $\zeta>2 a$. Considering the two cases of $l\left(p_{0}\right)>2 a$ and $l\left(p_{0}\right)<2 a$, one has the MTCS

$C_{\mathrm{sw}}=\pi a^{2}\left[\frac{2}{3} X U(1-X)+\left(1-\frac{1}{3 X^{2}}\right) U(X-1)\right], X=\frac{2 a}{l\left(p_{0}\right)}$,

which is identical to Eq. (16) of Minato et al. (2004).

\section{MTCS of aggregates}

\subsection{Model aggregates}

We take two kinds of model dust aggregates BPCAs and BCCAs (e.g., Mukai et al. 1992). Typical dust aggregates of BPCA and BCCA are shown in Fig. 2. The BPCAs are rather compact aggregates formed via ballistic particle-cluster aggregations, whereas the BCCAs are sparse ones formed via ballistic cluster-cluster aggregations. The fractal dimension is $D \simeq 3$ for the BPCAs and $D \simeq 2$ for the BCCAs (Meakin 1984; Mukai et al. 1992). For BCCAs and BPCAs composed of a large number of monomers $(N \gg 1)$, their geometrical cross sections $S_{\mathrm{z}}$ are related to the fractal dimension as

$D=\frac{\mathrm{d} \ln N}{\mathrm{~d} \ln \sqrt{\bar{S}_{z}}} \quad(N \gg 1)$,

where $\bar{S}_{z}$ is the geometrical cross section $S_{z}$ of the aggregate averaged over aggregate orientations (see Appendix A).

\subsection{Procedure for numerical calculations of the MTCS}

We calculate the MTCSs $\bar{C}_{\mathrm{sw}}$ averaged over aggregate orientations, namely,

$\bar{C}_{\mathrm{sw}}=\frac{1}{2 \pi} \int_{0}^{2 \pi} \mathrm{d} \phi \int_{0}^{\pi / 2} \mathrm{~d} \theta \sin \theta C_{\mathrm{sw}}(\theta, \phi)$,

where $\theta$ is the angle measured from the $z$ axis and $\phi$ the azimuth angle measured on the $x y$ plane from the $x$ axis in the counterclockwise direction. 


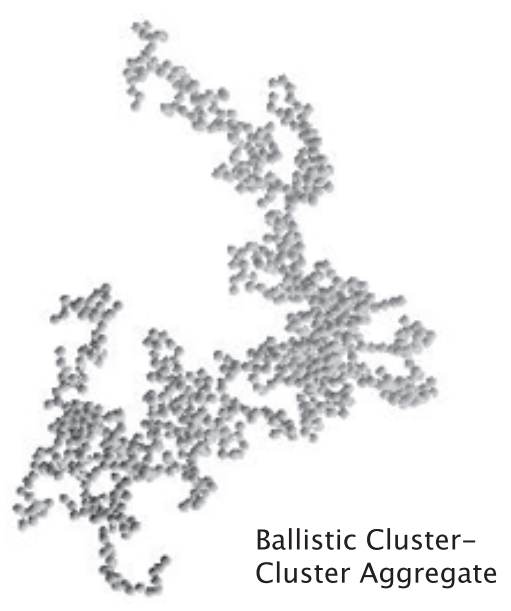

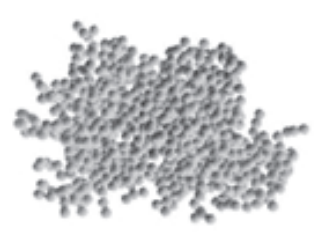

Ballistic ParticleCluster Aggregate

Fig. 2. Models of dust aggregates.

The numerical procedure is as follows:

1. Calculation of $\xi(x, y)$ :

We set a rectangular mesh of widths $\Delta x$ and $\Delta y$ in the $x y$ plane in the Cartesian coordinates. Each grid point is specified by $\left(x_{i}, y_{j}\right)$. For each grid, we calculate the thickness of the aggregate $\xi\left(x_{i}, y_{j}\right)$.

2. Calculation of $f(\zeta) \mathrm{d} \zeta$ :

We prepare a one-dimensional array $N_{k}=0\left(0 \leq k \leq k_{\max }\right)$. If $\xi_{i j} \equiv \xi\left(x_{i}, y_{j}\right)$ is $\zeta_{k}<\xi_{i j}<\zeta_{k+1}$, then we add one to the bin $N_{k}$. Here, we set $\zeta_{k+1}-\zeta_{k}=$ const $\equiv \Delta \zeta$. This procedure is done for all sets of $(i, j)$ and yields a set of $\left\{N_{k}\right\}=\left\{N_{1}, N_{2}, \cdots, N_{k_{\max }}\right\}$. The thickness distribution function is given by

$f\left(\zeta_{k}\right) \Delta \zeta=N_{k} \Delta \zeta$

The value of $k_{\max }$ must be equal to or larger than $\max \left\{\xi_{i j}\right\} / \Delta \zeta$.

3. Calculation of $C_{\mathrm{sw}}$ :

Using Eq. (6) and $f(\zeta) \Delta \zeta$, the MTCS is calculated as

$C_{\mathrm{sw}}=\frac{\Delta x \Delta y}{l\left(p_{0}\right)} \sum_{k=0}^{k_{1}} N_{k} \zeta_{k}+\Delta x \Delta y \sum_{k=k_{1}+1}^{k_{\max }} N_{k}$,

where $k_{1}$ is the bin number for which $\zeta_{k_{1}} \leq l\left(p_{0}\right)<\zeta_{k_{1}+1}$; we set $k_{1}=k_{\max }$ and take only the first term on the right-hand side if $\zeta_{k_{\max }}<l\left(p_{0}\right)$, which implies that all the incident ions penetrate through the aggregate.

4. Calculation of $\bar{C}_{\mathrm{sw}}$ :

Instead of rotating the aggregate, we vary the direction of incident stellar wind ions. The direction is specified by two angles $\theta$ and $\phi$. The average over the solid angle of incident ions is done for 40 directions in $\phi$ and 10 directions in $\theta$. From Eq. (11), we calculate $\bar{C}_{\mathrm{sw}}$ as

$$
\bar{C}_{\mathrm{sw}}=-\frac{\Delta \phi \Delta(\cos \theta)}{2 \pi} \sum_{i=1}^{10} \sum_{j=1}^{40} C_{\mathrm{sw}}\left(\cos ^{-1} \frac{i}{10}, 2 \pi \frac{j}{40}\right) .
$$

In the numerical calculations, we set $\Delta x=\Delta y=\Delta \zeta$ and make calculations for $1<N<2^{14}$ and $0.1<2 a / l\left(p_{0}\right)<5$, where $a$ is the monomer radius. To see the accuracy of the numerical calculations, we numerically calculate $C_{\mathrm{sw}}$ for spherical dust particles and compare with Eq. (9) by varying $\Delta x / a$ and the size parameter $X=2 a / l\left(p_{0}\right)$. The relative error is approximately proportional to $(\Delta x)^{2}$ and less than $10^{-3}$ for $\Delta x=2 a / 100$ in the size parameter range $X<5$. In what follows, we take $\Delta x=2 a / 100$.

\section{Results}

\subsection{Numerical computations for the MTCS}

Figure 3 shows the MTCSs averaged over orientations, $\bar{C}_{\mathrm{sw}}$, calculated numerically for the BPCAs and the BCCAs as a function of the number of monomers $N$. The cross section $\bar{C}_{\mathrm{sw}}$ is normalized by the geometrical cross sections $\pi a_{\mathrm{v}}^{2}$ of spheres with the same volumes as the aggregates, where $a_{\mathrm{v}}=N^{1 / 3} a$. In the cross section $\bar{C}_{\mathrm{sw}}$ of the aggregate, two effects appear: (1) the geometrical cross section $\bar{S}_{z}$ larger than that of a compact sphere; (2) the penetration of stellar wind ions through the aggregates (Minato et al. 2004). The first effect increases cross section $\bar{C}_{\mathrm{sw}}$, whereas the second one decreases $\bar{C}_{\mathrm{sw}}$. For an aggregate composed of a small number of monomers (i.e., small $N$ ) of small radius (i.e., $\left.a / l\left(p_{0}\right)<1\right), \bar{C}_{\mathrm{sw}}$ is less than the geometrical cross section $\pi a_{\mathrm{v}}^{2}$ of a sphere of the same volume as is seen from Fig. 3 . This is because the penetration effect dominates the increase in the geometrical cross section. For an aggregate of high $N$, on the other hand, $\bar{C}_{\mathrm{sw}}$ becomes larger than $\pi a_{\mathrm{v}}^{2}$ because of the effect in the increase in the geometrical cross section. For $N \gg 1$, the ratio $\bar{C}_{\mathrm{sw}} / \pi a_{\mathrm{v}}^{2}$ tends to a constant of $\bar{C}_{\mathrm{sw}} / \pi a_{\mathrm{v}}^{2} \simeq 4.5$ for BPCAs, while $\bar{C}_{\mathrm{sw}} / \pi a_{\mathrm{v}}^{2}$ tends to be proportional to $N^{1 / 3}$ for BCCAs. The behavior of $\bar{C}_{\text {sw }}$ for large $N$ can be understood in the following way. For $N \gg 1$, the cross section is approximated to be the geometrical cross section, i.e. $\bar{C}_{\mathrm{sw}} \sim \pi a^{2} N^{2 / D}$, where $D$ is fractal dimension of the aggregate. Since $\pi a_{\mathrm{v}}^{2}=\pi a^{2} N^{2 / 3}$, one has $\bar{C}_{\mathrm{sw}} / \pi a_{\mathrm{v}}^{2} \sim N^{2 / D-2 / 3}$ for $N \gg 1$. Thus, $\bar{C}_{\mathrm{sw}} / \pi a_{\mathrm{v}}^{2} \sim$ const for BPCAs for which $D \simeq 3$, and $\bar{C}_{\mathrm{sw}} / \pi a_{\mathrm{v}}^{2} \sim N^{1 / 3}$ for BCCAs for which $D \simeq 2$.

\subsection{An empirical formula for the MTCS}

We find that $\bar{C}_{\mathrm{sw}}$ are approximated well by an empirical formula similar to Eq. (9):

$\bar{C}_{\mathrm{sw}}=\bar{S}_{z}\left[\frac{2}{3} X^{\prime} U\left(1-X^{\prime}\right)+\left(1-\frac{1}{3 X^{\prime 2}}\right) U\left(X^{\prime}-1\right)\right]$,

where $X^{\prime}=3 V / 2 \bar{S}_{z} l\left(p_{0}\right)=\left(\pi a^{2} N / \bar{S}_{z}\right) X$ and $V=(4 / 3) \pi a^{3} N$. Equation (15) reduces to Eq. (9) for a spherical grain where $\bar{S}_{z}=\pi a^{2}$ and $X^{\prime}=X$. It is easily verified by similar calculations given in Sect. 2 for a spherical grain that Eq. (15) equals the MTCS of an oblate spheroid of semi-major axes of $\left(\bar{S}_{z} / \pi\right)^{1 / 2}$ and the semi-minor (symmetric) axis, which is parallel to the $z$ axis and equal to $3 V / 4 \bar{S}_{z}$. The cross section $\bar{C}_{\mathrm{sw}}$ calculated using 

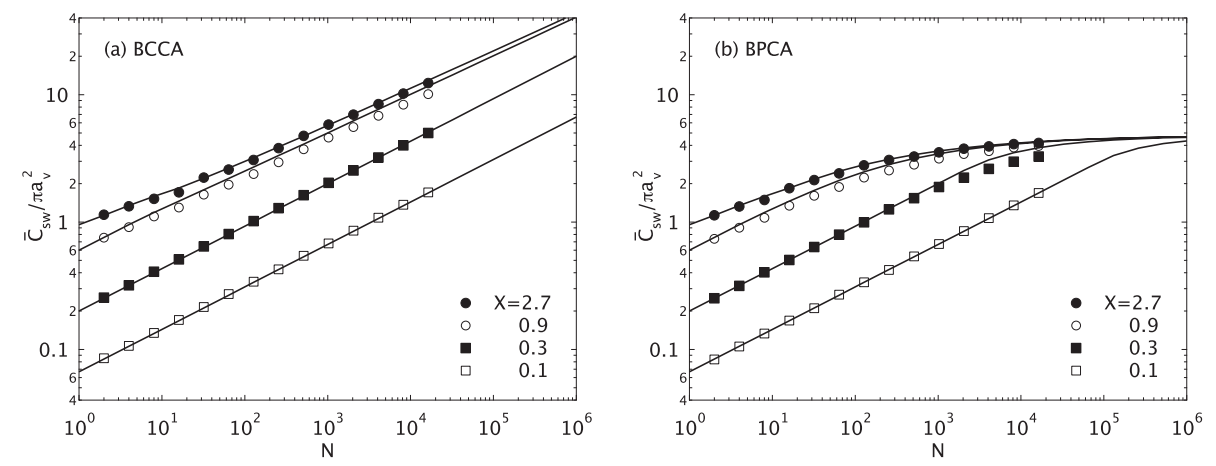

Fig. 3. Momentum transfer cross section averaged over aggregate orientations, $\bar{C}_{\mathrm{sw}}$, for ballistic cluster-cluster aggregates (BCCAs; left) and ballistic particle-cluster aggregates (BPCAs; right) versus the number of monomers $N$. The vertical axis shows $\bar{C}_{\mathrm{sw}}$ divided by the geometrical cross section of a sphere of the same volume as the aggregate. The lines indicate the cross section given by the empirical formula Eq. (15). In the figure, $a_{\mathrm{v}}=N^{1 / 3} a$ is the radius of volume-equivalent spheres and $X=2 a / l\left(p_{0}\right)$ is the size parameter of monomers, where $a$ is the monomer radius and $l\left(p_{0}\right)$ is the projected range of stellar wind ions.

Eq. (15) is indicated by solid lines in Fig. 3. Equation (15) reproduces the numerical results for $1 \leq N \leq 2^{14}$ within an error of at most $20 \%$. For $a>0.01 \mu \mathrm{m}$ (i.e., $X \geq 0.3$ ), our numerical computations show that the MTCS $\bar{C}_{\text {sw }}$ is approximated by the geometrical cross section $\bar{S}_{z}$ as $N$ increases. This indicates that all the incident ions are absorbed by large aggregates. At $N>2^{14}$, the MTCS $\bar{C}_{\mathrm{sw}}$ should be the geometrical cross section $\bar{S}_{z}$, because all the incident ions are absorbed as well. Equation (15) is valid even for $N>2^{14}$ because the equation gives $\bar{C}_{\mathrm{sw}}=\bar{S}_{z}$ at sufficiently large $N$. We will use Eq. (15) in the following calculations, instead of numerical values for $\bar{C}_{\mathrm{sw}}$.

\subsection{Radial pressure on dust aggregates}

We consider solar wind as an example of stellar winds. We use the same solar-wind parameters as those used in Minato et al. (2004): $v_{\mathrm{sw}}=4.5 \times 10^{7} \mathrm{~cm} \mathrm{~s}^{-1}$ for the radial velocity, $n(r)=n_{0}(r / \mathrm{AU})^{-2}$ with $n_{0}=6 \mathrm{~cm}^{-3}$ for the radial density distribution, and the number ratio for protons and $\alpha$ particles to be $1: 0.05$, while neglecting heavier particles (Phillips et al. 1995; Neugebauer 2001). These parameter values reflect ecliptic conditions, which are appropriate for the majority of interplanetary dust.

We take silicate $\left(\mathrm{MgFeSiO}_{4}\right)$ and carbon as plausible dust materials. The data of the stopping power of carbon used here are the same as in Minato et al. (2004). For silicate, for which there are no data, we calculate the stopping power with the use of the Bragg's rule (Bragg \& Kleeman 1905). This rule estimates the stopping power as a sum of the stopping powers of atoms composing the solid. The error of the Bragg's rule for $\mathrm{SiO}_{2}$ is less than $50 \%$ for protons of energies $1-10 \mathrm{keV}$ (Bauer et al. 1998). Table 1 lists the projected ranges and stopping cross sections of protons and $\alpha$-particles with initial kinetic energy $1 \mathrm{keV} / \mathrm{amu}$ for silicate $\left(\mathrm{MgFeSiO}_{4}\right)$ and carbon.

Figure 4 shows the ratio $\beta_{\mathrm{sw}}$ of solar wind pressure to gravitational attraction acting on dust particles around the sun. The $\beta_{\mathrm{sw}}$ values are much smaller than unity and thus the solar wind pressure is negligibly small compared to the gravitational attraction. For a star of mass-loss rate $\dot{M}$, the $\beta_{\mathrm{sw}}$-value is scaled by multiplying $\dot{M} / \dot{M}_{\odot}$, if the energy distribution of the stellar wind is similar to that of the sun, where $\dot{M}_{\odot}$ is the mass loss rate of the sun. The stellar wind pressure is ineffective unless $\dot{M}>10^{3} \dot{M}_{\odot}$, which is implausible even for young solar-like stars (Wood et al. 2005).
Table 1. The projected range $l_{i}$ and the stopping cross section $S_{i}^{(1)}$ of protons $(i=\mathrm{p})$ and $\alpha$-particles $(i=\alpha)$ with initial kinetic energy $1 \mathrm{keV} / \mathrm{amu}$ for silicate $\left(\mathrm{MgFeSiO}_{4}\right)$ and carbon.

\begin{tabular}{lcccc}
\hline \hline & $\begin{array}{c}l_{\mathrm{p}} \\
{[\mu \mathrm{m}]}\end{array}$ & $\begin{array}{c}l_{\alpha} \\
{[\mu \mathrm{m}]}\end{array}$ & $\begin{array}{c}S_{\mathrm{p}}^{(1)} \\
{\left[10^{-15} \mathrm{eV} \mathrm{cm}^{2}\right]}\end{array}$ & $\begin{array}{c}S_{\alpha}^{(1)} \\
{\left[10^{-15} \mathrm{eV} \mathrm{cm}^{2}\right]}\end{array}$ \\
\hline Silicate & 0.071 & 0.17 & $25^{a}$ & $40^{a}$ \\
Carbon & 0.067 & 0.14 & $3.0^{b}$ & $5.9^{c}$ \\
\hline
\end{tabular}

References: ${ }^{a}$ Ziegler (2002); ${ }^{b}$ Møller et al. (2002); ${ }^{c}$ Mikheev et al. (1993).

\subsection{Tangential drag on dust aggregates}

The ratio of the plasma PR drag to the photon PR drag is given by

$\gamma=\frac{\bar{C}_{\mathrm{sw}}}{\bar{C}_{\mathrm{ph}}} \frac{\dot{M} c^{2}}{L}=0.3\left(\frac{\bar{C}_{\mathrm{sw}}}{\bar{C}_{\mathrm{ph}}}\right)\left(\frac{\dot{M}}{\dot{M}_{\odot}}\right)\left(\frac{L}{L_{\odot}}\right)^{-1}$,

where $L$ is a stellar luminosity and $\bar{C}_{\mathrm{ph}}$ is the radiation pressure cross section averaged over the orientation of a dust aggregate and the spectrum of stellar radiation. The ratio of stellar wind pressure to radiation pressure is much lower than unity because the momentum flux of stellar photons is much larger than that of the stellar wind. On the other hand, the aberration angle of stellar photons is much smaller than the incident angle of stellar wind to the aggregate orbiting a star because the stellar wind speed is much slower than the speed of light. As a consequence, $\gamma$ is on the order of unity for $\dot{M} \sim \dot{M}_{\odot}$ and $L \sim L_{\odot}$, implying that the plasma PR drag plays a role.

The ratio $\gamma$ is shown in Fig. 5 for silicate aggregates and carbon aggregates orbiting the sun. The radius of their monomers is set to $a=0.1 \mu \mathrm{m}$. The right axis indicates the ratio of solar wind pressure $\beta_{\mathrm{sw}}$ to solar radiation pressure $\beta_{\mathrm{ph}}$. Calculations of the radiation pressure $\beta_{\mathrm{ph}}$ will be given elsewhere. Figure 5 indicates that the plasma PR effect is dominant or comparable to the photon PR effect. The $\gamma$ values for carbon are slightly lower than those for silicate. The PR drags may not be relevant to carbon aggregates consisting of a small number of monomers since they would be easily blown out of the system by solar radiation. Note that the ratio $\gamma$ for the aggregates is always higher than the one for the spherical particle of equal volume. This indicates that the plasma PR drag is more effective for the aggregates than for the spherical particles of equal volume. It should be noted that, for dust aggregates around young stars, the plasma PR effect exceeds the photon PR effect because $\dot{M} \gg \dot{M}_{\odot}$ and $L \sim L_{\odot}$. 

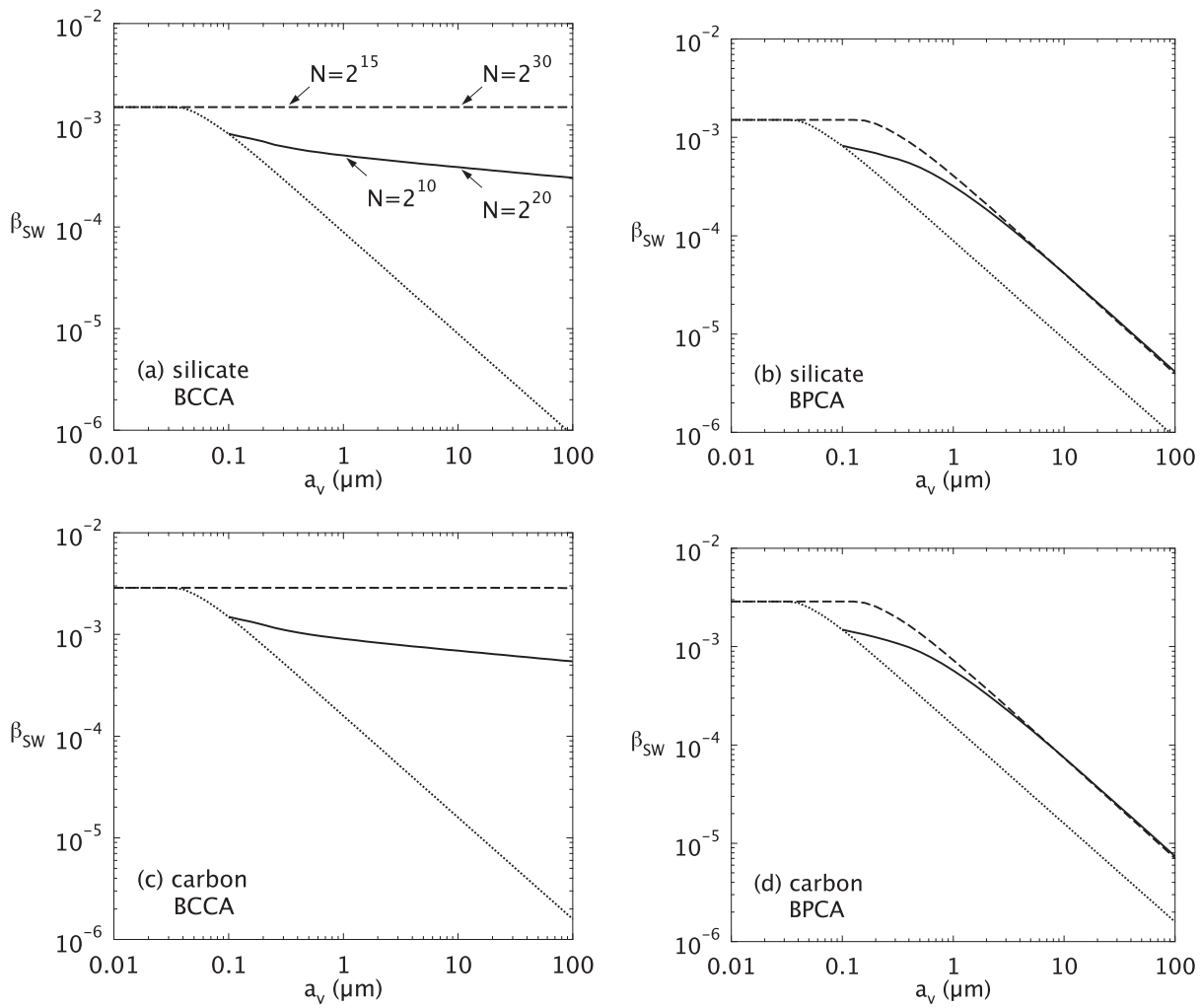

Fig. 4. The ratio $\beta_{\mathrm{sw}}$ of the solar wind pressure to gravitational attraction by the sun for aggregates: a) silicate BCCAs (ballistic cluster-cluster aggregates), b) silicate BPCAs (ballistic particle-cluster aggregates), c) carbon BCCAs, d) carbon BPCAs. The solid and dashed curves are the $\beta_{\mathrm{sw}}$ ratios with the monomer radius of 0.1 and $0.01 \mu \mathrm{m}$, respectively. The dotted line indicates the ratio $\beta_{\mathrm{sw}}$ for a spherical dust taking into account the effect of passage of incident ions through the dust grains. Here, $a_{\mathrm{v}}$ is the radius of volume-equivalent spheres.
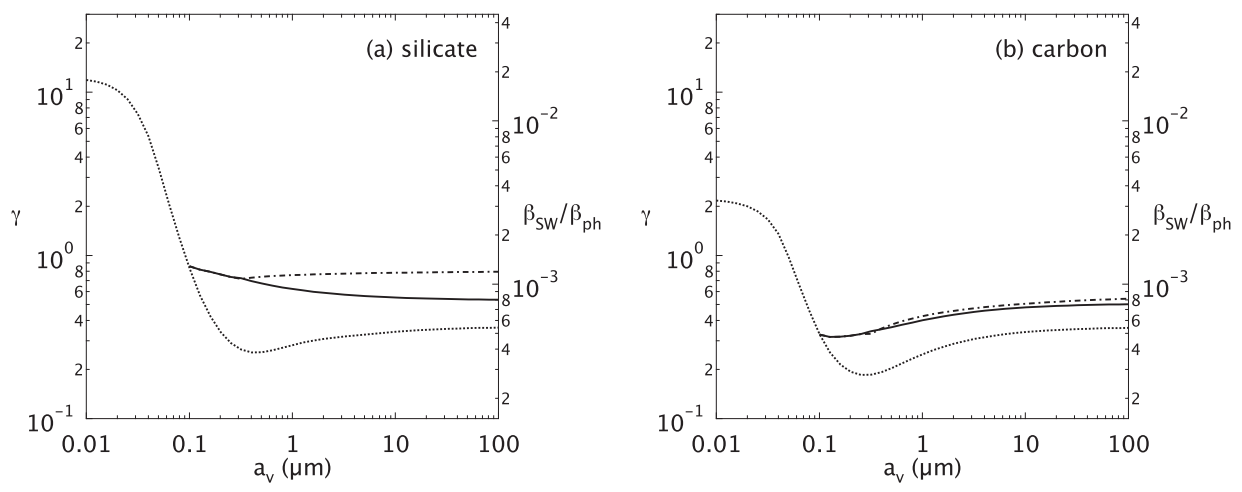

Fig. 5. Ratio $\gamma$ of the plasma PR drag to the photon PR drag for silicate and carbon aggregates consisting of $0.1 \mu$ m-radius monomers. The right axis indicates the ratio of the stellar wind pressure to the radiation pressure. Solid, dash-dotted, and dotted lines are the results for ballistic particle-cluster aggregates (BPCAs), ballistic cluster-cluster aggregates (BCCAs), and spheres, respectively.

Figure 6 shows the time required for dust aggregates consisting of $0.1 \mu \mathrm{m}$ monomers at $50 \mathrm{AU}$ from the central star to fall to the star due to the plasma PR effect. The falling time for a spherical grain is shown for comparison. Also shown is the falling time for a spherical grain due to the photon PR effect. Figure 6 shows that the falling time is shorter for the aggregates than that for a spherical particle because the ratio $\gamma$ is higher for the aggregates than for the spherical grains. It should be noted that, for stars with high mass loss rate and luminosity of $\sim 1 L_{\odot}$ such as young main-sequence stars, the falling time due to the plasma PR drag is shorter by orders of magnitude than the one caused by the photon PR drag for any size. This is more pronounced if the monomer size is smaller than $0.1 \mu \mathrm{m}$.

\section{Discussion}

In the zodiacal dust cloud of our own solar system, mutual collisions have a negligible effect compared to the photon PR effect, except for very large grains of mass greater than $10^{-8} \mathrm{~kg}$ (Grün et al. 1985). Under the current solar-wind condition, the plasma PR effect is known to be comparable to the photon PR effect (Mukai \& Yamamoto 1982). We have shown that the fluffy structure of aggregates enhances the relative importance of the plasma PR drag. This drag becomes even stronger than the photon PR drag for the aggregates composed of small monomers $(a \sim 0.01 \mu \mathrm{m})$. For the young sun, the mass loss rate is estimated to be two orders of magnitude higher than for the present sun, while the luminosity does not change significantly with time 

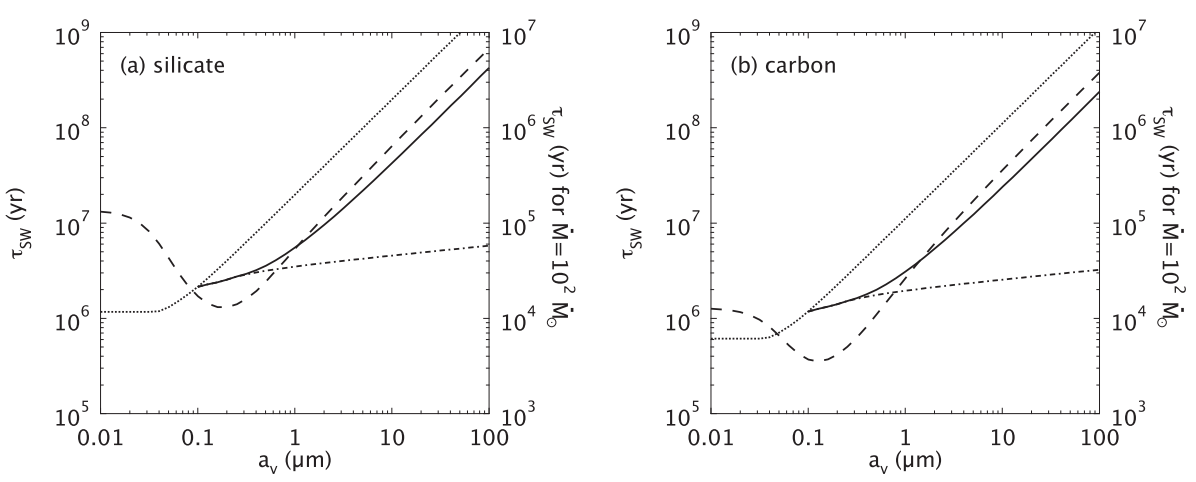

Fig. 6. Falling time for ballistic particle-cluster aggregates (BPCAs; solid lines) and ballistic cluster-cluster aggregates (BCCA; dash-dotted lines) and for spherical particles (dotted lines) from $50 \mathrm{AU}$ from a central star. The left and right axes indicate the falling times for the mass loss rates of 1 and $100 \dot{M}_{\odot}$, respectively. The dashed line is the falling time for spherical particles due to the photon PR effect under the solar luminosity of $1 L_{\odot}$ (see the left axis). Note that the range of luminosities of young main-sequence stars is limited around $\sim 1 L_{\odot}$.

(Wood et al. 2002, 2005). As a consequence, the plasma PR effect should have been the dominant process in removing dust particles orbiting the young sun (e.g., $t \leq 1 \mathrm{Gyr}$ ). The result of the short falling time due to the plasma PR effect requires a high dust production rate to maintain the zodiacal dust cloud around the young sun.

We make a sketch for the dominant regions of the plasma PR drag, the photon PR drag, and the mutual collisions in terms of the mass loss rate of a star and the total dust mass in its circumstellar disk (see Fig. B.1). In massive debris disks like Vegatype stars, collisional destruction plays a major role in removing dust particles from the disks, owing to their high concentrations in space. The collisional lifetimes for dust in the observed debris disks are typically four orders of magnitude shorter than their photon PR lifetimes (Dominik \& Decin 2003). The plasma PR effect is also negligible for the removal of dust particles in the massive disks, unless the flux ratio of the stellar wind to the stellar radiation exceeds ten thousand times the ratio for the sun. Observations show that the total dust mass $M_{\mathrm{d}}$ decreases with the ages of stars, thus dust particles are less frequently destroyed by collisions at the late stages of the disk evolution (e.g., Metchev et al. 2004). Because collisional lifetimes are inversely proportional to the total disk dust mass (see Eq. (B.3)), the total dust mass will reach a value in which the collisional destruction no longer plays a role compared to the photon PR effects. Even at such late stages of the disk evolution, the plasma PR effect might still be insignificant because the mass loss rate of the star also decreases with the stellar age. However, there should be main-sequence stars whose disk luminosities are so low that observations with currently available instruments have not been successful enough so far to detect. For low-mass disks around young stars, we expect the near-future detection of such faint dust clouds where the collisional destruction is insignificant and the plasma PR effect may dominate the dust removal process.

Acknowledgements. We would like to thank Munetaka Ueno and Brian E. Wood for useful communications. This research is supported by the German Aerospace Center DLR (Deutschen Zentrum für Luft- und Raumfahrt) under the projects "Kosmischer Staub: Der Kreislauf interstellarer und interplanetarer Materie" (RD-RX-50 OO 0101-ZA), "Mikro-Impakte" (RD-RX-50 OO 0203), "Rosetta: MIDAS, MIRO, MUPUS” (RD-RX-50 QP 0403), and by MEXT Japan, Grantin-Aid for Scientific Research on Priority Areas, "Development of Extra-Solar Planetary Science" (\#16077203).

\section{Appendix A: Empirical formulae for the geometrical cross sections of dust aggregates}

For practical purposes, we give empirical formulae for $\bar{S}_{z}$, the geometrical cross sections of the BPCAs and BCCAs averaged

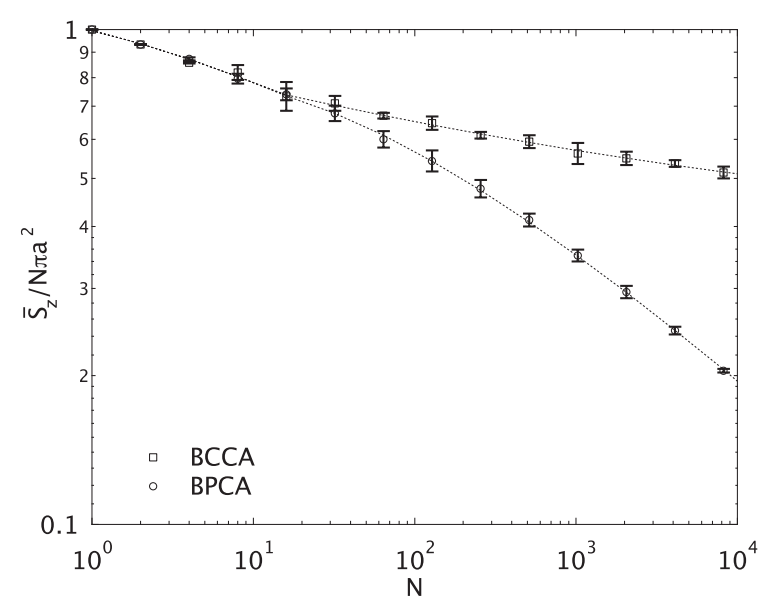

Fig. A.1. Geometrical cross sections of aggregates, $\bar{S}_{z}$, relative to the sum of monomer cross sections, $N \pi a^{2}$. Mean values are plotted by symbols, their standard deviations are given by error bars, and fitting curves are indicated by dotted lines. Squares: ballistic cluster-cluster aggregates (BCCAs); Circles: ballistic particle-cluster aggregates (BPCAs).

over their orientations. Figure A. 1 shows $\bar{S}_{z} /\left(N \pi a^{2}\right)$ for three aggregates formed numerically by random ballistic particle-cluster or cluster-cluster collisions. By making a fit to $\bar{S}_{z}$ 's, we obtain the following empirical formula for $\bar{S}_{z}$. For BPCAs, the $\bar{S}_{z}$ is approximated by

$\frac{\bar{S}_{z}}{\pi a^{2} N}= \begin{cases}4.27 N^{-0.315} \exp \left(-1.74 / N^{0.243}\right) & (N \geq 16) \\ 12.5 N^{-0.315} \exp \left(-2.53 / N^{0.0920}\right) & (N<16)\end{cases}$

For BCCAs, the $\bar{S}_{z}$ is given by

$$
\frac{\bar{S}_{z}}{\pi a^{2} N}= \begin{cases}0.352+0.566 N^{-0.138} & (N \geq 16) \\ 12.5 N^{-0.315} \exp \left(-2.53 / N^{0.0920}\right) & (N<16)\end{cases}
$$

\section{Appendix B: Lifetimes of dust particles}

We give the expressions of three timescales that determine the lifetime of dust in a debris dust disk around a star. The lifetime of a dust particle against the plasma PR effect falling from a distance $r$ to the central star is expressed by

$$
\begin{aligned}
\tau_{\mathrm{sw}}= & 6 \times 10^{6} \mathrm{yr}\left(\frac{r}{50 \mathrm{AU}}\right)^{2} \\
& \times\left(\frac{a_{\mathrm{v}}}{\mu \mathrm{m}}\right)\left(\frac{\rho_{\mathrm{d}}}{\mathrm{g} \mathrm{cm}^{-3}}\right)\left(\frac{\pi a_{\mathrm{v}}^{2}}{\bar{C}_{\mathrm{sw}}}\right)\left(\frac{\dot{M}}{\dot{M}_{\odot}}\right)^{-1},
\end{aligned}
$$




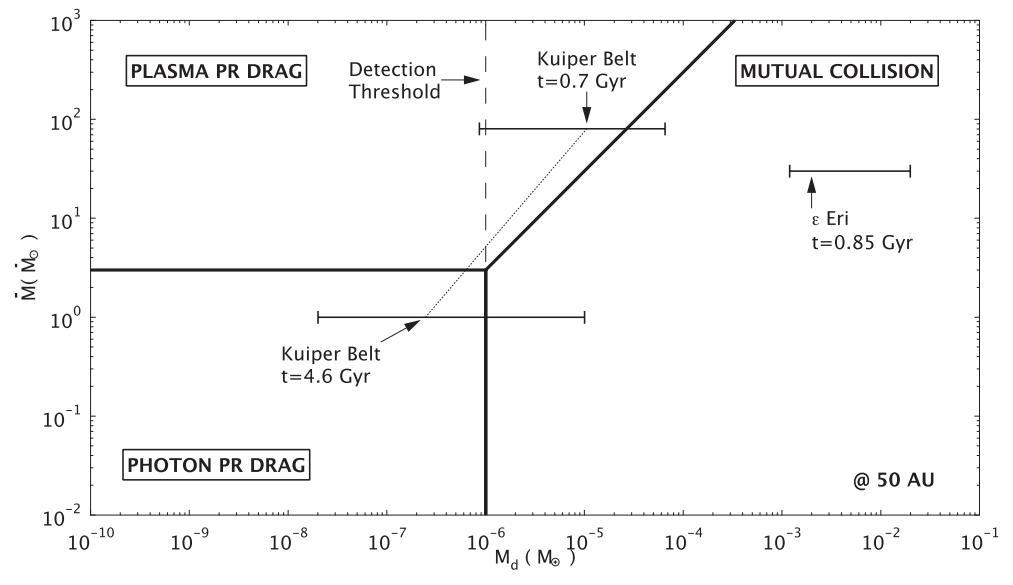

Fig. B.1. Dust-removal processes in the phase space for the mass loss rate of a star $\dot{M}$ and the total dust mass of its circumstellar disk $M_{\mathrm{d}}$. The dominant process is determined by the shortest lifetime among Eqs. (B.1)-(B.3).

where $a_{\mathrm{v}}$ and $\rho_{\mathrm{d}}$ are the volume-equivalent sphere radius and bulk density of a dust particle, respectively. On the other hand, the lifetime against the photon PR effect is given by, for example, Burns et al. (1979):

$$
\begin{aligned}
\tau_{\mathrm{ph}}= & 2 \times 10^{6} \mathrm{yr}\left(\frac{r}{50 \mathrm{AU}}\right)^{2} \\
& \times\left(\frac{a_{\mathrm{v}}}{\mu \mathrm{m}}\right)\left(\frac{\rho_{\mathrm{d}}}{\mathrm{g} \mathrm{cm}^{-3}}\right)\left(\frac{\pi a_{\mathrm{v}}^{2}}{\bar{C}_{\mathrm{ph}}}\right)\left(\frac{L}{L_{\odot}}\right)^{-1},
\end{aligned}
$$

where $L$ and $L_{\odot}$ are the luminosities of the star and the sun, respectively, and $\bar{C}_{\mathrm{ph}}$ is the cross section for radiation pressure. The collisional lifetime of dust due to collisions with other dust particles in the collision cascade is given by Najita \& Williams (2005):

$$
\begin{aligned}
\tau_{\text {coll }}= & 2 \times 10^{3} \mathrm{yr}\left(\frac{r}{50 \mathrm{AU}}\right)^{7 / 2} \\
& \times\left(\frac{a_{\mathrm{v}}}{\mu \mathrm{m}}\right)\left(\frac{\rho_{\mathrm{d}}}{\mathrm{g} \mathrm{cm}^{-3}}\right)\left(\frac{\pi a_{\mathrm{v}}^{2}}{\bar{S}_{z}}\right)\left(\frac{M}{M_{\odot}}\right)^{-1 / 2}\left(\frac{M_{\mathrm{d}}}{10^{-3} M_{\oplus}}\right)^{-1},
\end{aligned}
$$

where $M_{\mathrm{d}}, M, M_{\odot}$, and $M_{\oplus}$ are the masses of the dust disk, the central star, the sun, and the earth, respectively.

The dominant process is determined by the shortest lifetime among Eqs. (B.1)-(B.3). Figure B.1 illustrates the dominant regions of the above-mentioned processes in the phase space for the total dust mass and the stellar mass loss rate. The boundaries of the three dominant processes are determined by equating Eqs. (B.1)-(B.3). Note that the boundaries are independent of $a_{\mathrm{v}}$ and $\rho_{\mathrm{d}}$, although the absolute values of $\tau_{\mathrm{sw}}, \tau_{\mathrm{ph}}$, and $\tau_{\text {coll }}$ depend on them. Nominal values such as $r=50 \mathrm{AU}$ and the assumption of $\bar{C}_{\mathrm{sw}} \approx \bar{C}_{\mathrm{pr}} \approx \bar{S}_{z}$ are used to determine the boundaries among the processes. The figure shows the dominant process for dust around the sun and $\epsilon$ Eridani whose mass loss rate is given by Wood et al. $(2002,2005)$. The total mass of dust in the Kuiper belt is estimated by Backman et al. (1995) and by Yamamoto \& Mukai (1998), who include production of dust particles by interstellar dust impacts on Kuiper belt objects. When the sun was young at its $t=0.7 \mathrm{Gyr}$ age, both the mass loss rate and the total dust mass were higher than the current values as expected from the mass loss history of solar-like stars $\left(\dot{M} \propto t^{-2.33}\right)$ given by Wood et al. (2005) and the collisional evolution $\left(\dot{M}_{\mathrm{d}} \propto t^{-1}\right)$ or PR drag evolution $\left(\dot{M}_{\mathrm{d}} \propto t^{-2}\right)$ of dust given by Dominik \& Decin (2003). The total dust mass of $\epsilon$ Eri varies from one estimate to another within the range for typical debris disks (Greaves et al. 1998 2005; Schütz et al. 2004; Sheret et al. 2004). Also plotted in Fig. B.1 as a dashed line is the detection threshold of currently available top-class instruments (see, e.g., Metchev et al. 2004; Beichman et al. 2005).

\section{References}

Backman, D. E., Dasgupta, A., \& Stencel, R. E. 1995, ApJ, 450, L35 Bauer, P., Golser, R., Semrad, D., et al. 1998, Nucl. Inst. Methods Phys. Res. B, 136, 103

Beichman, C. A., Bryden, G., Rieke, G. H., et al. 2005, ApJ, 622, 1160

Bragg, W. H., \& Kleeman, R. 1905, Phil. Mag., 10, 53

Brownlee, D. E. 1978, in Cosmic Dust, ed. J. A. M. McDonnell (New York: Wiley-Interscience), 295

Burns, J. A., Lamy, P. L., \& Soter, S. 1979, Icarus, 40, 1

Dominik, C., \& Decin, G. 2003, ApJ, 598, 626

Fahr, H. J., Scherer, K., \& Banaszkiewicz, M. 1995, Planet. Space Sci., 43, 301

Fermi, E., \& Teller, E. 1947, Phys. Rev., 72, 399

Fixsen, D. J., \& Dwek, E. 2002, ApJ, 578, 1009

Greaves, J. S., Holland, W. S., Moriarty-Schieven, G., et al. 1998, ApJ, 506, L133

Greaves, J. S., Holland, W. S., Wyatt, M. C., et al. 2005, ApJ, 619, L187

Grün, E., Zook, H. A., Fechtig, H., \& Giese, R. H. 1985, Icarus, 62, 244

Gustafson, B. Å. S. 1994, Annu. Rev. Earth Planet. Sci., 22, 553

Lindhard, J., Scharff, M., \& Schiott 1963, Mathematisk-fysiske Meddelelser det Kongelige Danske Vindenskarbernes Selskab, 33, No. 14

Meakin, P. 1984, Phys. Rev. A, 29, 997

Metchev, S. A., Hillenbrand, L. A., \& Meyer, M. R. 2004, ApJ, 600, 435

Mikheev, S., Ryzhov, Y., Shkarban, I., \& Yurasova, V. 1993, Nucl. Instrum. Methods Phys. Res. B, 78, 86

Minato, T., Köhler, M., Kimura, H., Mann, I., \& Yamamoto, T. 2004, A\&A, 424, L13

Møller, S. P., Csete, A., Ichioka, T., et al. 2002, Phys. Rev. Lett., 88, 193201

Mukai, T., \& Yamamoto, T. 1982, A\&A, 107, 97

Mukai, T., Ishimoto, H., Kozasa, T., Blum, J., \& Greenberg, J. M. 1992, A\&A, 262,315

Mukai, T., Higuchi, A., Lykawka, P. S., et al. 2004, Adv. Space Res., 34(1), 172 Najita, J., \& Williams, J. P. 2005, ApJ, 635, 625

Neugebauer, M. 2001, in The Heliosphere Near Solar Minimum, ed. A. Balogh, R. G. Marsden, \& E. J. Smith (Chichester: Springer)

Plavchan, P., Jura, M., \& Lipscy, S. J. 2005, ApJ, 631, 1161

Phillips, J. L., Bame, S. J., Barnes, A., et al. 1995, Geophys. Res. Lett., 22, 3301

Schütz, O., Nielbock, M., Wolf, S., Henning, T., \& Els, S. 2004, A\&A, 414, L9

Sheret, I., Dent, W. R. F., \& Wyatt, M. C. 2004, MNRAS, 348, 1282

Sigmund, P., \& Schinner, A. 2002, Nucl. Instr. Methods Phys. Res. B., 193, 49

Wood, B. E., Müller, H.-R., Zank, G. P., \& Linsky, J. L. 2002, ApJ, 574, 412

Wood, B. E., Müller, H.-R., Zank, G. P., Linsky, J. L., \& Redfield, S. 2005, ApJ, $628, \mathrm{~L} 143$

Yamamoto, S., \& Mukai, T. 1998, Earth Planets Space, 50, 531

Ziegler, J. F. 2002, The Stopping and Range of Ions in Matter: Plots of SRIM2003 Calculations and Experimental Results, http://www . srim.org/SRIM/SRIMPICS/STOPPLOTS.htm 